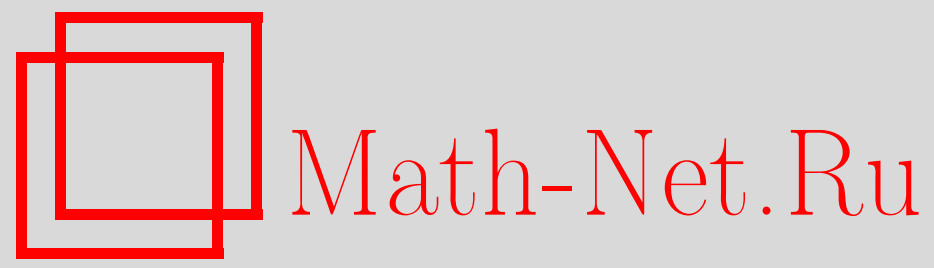

И. А. Дрожжин, Аналог теоремы Маркова, Вестник ТвГУ. Серия: Прикладная математика, 2017, выпуск 4, 73-83

DOI: https://doi.org/10.26456/vtpmk190

Использование Общероссийского математического портала Math-Net.Ru подразумевает, что вы прочитали и согласны с пользовательским соглашением

http://www.mathnet.ru/rus/agreement

Параметры загрузки:

IP: 35.173 .137 .237

26 апреля 2023 г., 17:19:43 


\section{ВЕЩЕСТВЕННЫЙ, КОМПЛЕКСНЫЙ И ФУНКЦИОНАЛЬНЫЙ АНАЛИЗ}

УДК 517.5

АНАЛОГ ТЕОРЕМЫ МАРКОВА

Дрожжин И.А.

Тверской государственный университет, г. Тверь

Поступила в редакцию 16.06.2017, после переработки 17.0\%.201\%.

В пространстве суммируемых на отрезке функций получен признак наилучшего приближения при аппроксимации элементами конечномерного конуса, который является аналогом известной теоремы Маркова A.A.

Ключевые слова: банахово пространство, замкнутое выпуклое множество, суммируемая функция, наилучшее приближение, конус конечной размерности, расширенный конус, интерполяционный полином.

Вестник ТвГУ. Серия: Прикладная математика. 2017. № 4. С. 73-83. https://doi.org/10.26456/vtpmk190

\section{Введение}

В пространстве суммируемых на отрезке функций рассматривается задача наилучшего приближения при аппроксимации элементами конуса конечной размерности. Приводятся признаки наилучшего приближения, в частности, в терминах расширенного конуса. Получен достаточный признак наилучшего приближения, который является аналогом теоремы Маркова А.А.. В конце работы рассматриваются известные способы построения наилучших приближений при аппроксимации подпространствами тригонометрических и алгебраических полиномов, которые могут быть использованы и в случае приближения элементами конуса с указанными образующими.

\section{1. Признаки наилучшего приближения в пространстве суммируемых функций}

Пусть $X$ - некоторое банахово пространство, $F$ - выпуклое замкнутое множество в $X$. Под задачей наилучшего приближения элемента $x \in X$ элементами множества $F$ будем понимать задачу отыскания величины

$$
E(x, F)=\inf \{\|x-h\|: h \in F\} .
$$


Число $E(x, F)$ назовем величиной наилучшего приближения элемента $x \in X$ элементами множества $F$, а элемент $u \in F$, для которого

$$
E(x, F)=\|x-u\|,
$$

назовем элементом наилучшего приближения (э.н.п.) для $x$ в $F$.

Геометрически наилучшее приближение элемента $x$ трактуется как его расстояние до множества $F$, а элемент наилучшего приближения - как точка множества $F$, ближайшая к $x$.

В качестве пространства $X$ рассмотрим банахово пространство $L[a ; b]$ вещественных функций, суммируемых на отрезке $[a ; b]$, с нормой

$$
\|x\|=\int_{a}^{b}|x(t)| d t, \quad x \in L[a ; b]
$$

Пусть $F-$ замкнутое выпуклое множество в $L[a ; b]$.

В [1] доказана

Теорема 1.1. Для того чтобы элемент $h_{0} \in F$ являлся э.н.п. для $x \in L[a ; b] \backslash F$ во множестве $F$ достаточно, а если мера множества

$$
\left\{t \in[a ; b]: x(t)=h_{0}(t)\right\}
$$

равна нулю, то и необходимо, выполнение следующего условия

$$
\int_{a}^{b}\left(h(t)-h_{0}(t)\right) \operatorname{sign}\left(x(t)-h_{0}(t)\right) d t \leq 0 \text { для любого } h \in F .
$$

Рассмотрим случай, когда замкнутое выпуклое множество $F$ является конечномерным конусом.

Пусть $\left\{\varphi_{1}, \varphi_{2}, \ldots, \varphi_{n}\right\}$ - линейно независимая система в $L[a ; b], m-$ целое неотрицательное число. Положим

$$
\begin{gathered}
H=\left\{\sum_{k=1}^{n} \alpha_{k} \varphi_{k}: \alpha_{k} \in R, \alpha_{j} \geq 0 \text { при } j>m\right\}, \\
h_{0}=\sum_{k=1}^{n} \lambda_{k} \varphi_{k} \in H, \\
J\left(h_{0}\right)=\{1,2, \ldots, m\} \cup\left\{k>m: \lambda_{k}>0\right\}, \\
H_{J\left(h_{0}\right)}=\left\{\sum_{k=1}^{n} \beta_{k} \varphi_{k}: \beta_{k} \in R, \beta_{j} \geq 0 \text { при } j \notin J\left(h_{0}\right)\right\} .
\end{gathered}
$$

Множества $H, H_{J\left(h_{0}\right)}$ являются замкнутыми конусами, при этом $H \subset H_{J\left(h_{0}\right)}$. Конус $H_{J\left(h_{0}\right)}$ в дальнейшем будем называть расширенным [4] относительно элемента $h_{0} \in H$. Функции $\varphi_{1}, \varphi_{2}, \ldots, \varphi_{n}$ назовем образующими конуса $H$. 
Лемма 1.1. Для любого $h_{0} \in H$ имеет место равенство

$$
H_{J\left(h_{0}\right)}=\left\{u \in L[a ; b]: u=\alpha\left(h-h_{0}\right), \alpha \geq 0, h \in H\right\} .
$$

\section{Доказательство.}

$$
\left\{u \in L[a ; b]: u=\alpha\left(h-h_{0}\right), \alpha \geq 0, h \in H\right\} \subset H_{J}\left(h_{0}\right)
$$

очевидно, т.к. элемент $h-h_{0} \in H_{J\left(h_{0}\right)}$ для любого $h \in H$ и следовательно

$$
\alpha\left(h-h_{0}\right) \in H_{J}\left(h_{0}\right)
$$

при $\alpha \geq 0$. Покажем, что

$$
H_{J\left(h_{0}\right)} \subset\left\{u \in L[a ; b]: u=\alpha\left(h-h_{0}\right), \alpha \geq 0, h \in H\right\} .
$$

Пусть $w \in H_{J\left(h_{0}\right)}$. Элемент $w$ можно представить в виде

$$
w=w+h_{0}-h_{0} .
$$

Подберем $m_{0} \in N$ таким образом, чтобы

$$
\frac{1}{m_{0}} w+h_{0} \in H
$$

Поэтому

$$
w=m_{0}\left(\frac{1}{m_{0}} w+h_{0}-h_{0}\right)=m_{0}\left(v-h_{0}\right),
$$

где $v=\frac{1}{m_{0}} w+h_{0} \in H$ и включение доказано. Лемма верна.

Так как конус $H$ является замкнутым выпуклым локально компактным множеством, то для каждого $x \in L[a ; b]$ существует э.н.п. в конусе $H$, а множество его элементов наилучшего приближения выпукло [3].

Следствие 1.1. Пусть $x \in L[a ; b] \backslash H, h_{0} \in H$. Для того чтобы $h_{0}$ был э.н.п. для $x$ при аппроксимации элементами конуса $H$, достаточно, а в случае, когда множество

$$
\left\{t \in[a ; b]: x(t)=h_{0}(t)\right\}
$$

имеет меру нуль и необходимо, выполнение условия

$$
\int_{a}^{b} \varphi(t) \operatorname{sign}\left(x(t)-h_{0}(t)\right) d t \leq 0
$$

для любого $\varphi \in H_{J\left(h_{0}\right)}$.

Для установления справедливости следствия достаточно применить лемму 1.1 о структуре расширенного конуса.

Сформулируем без доказательства критерий наилучшего приближения в терминах расширенного конуса. 
Теорема 1.2. Пусть $x \in L[a ; b]$. Элемент $h_{0}$ есть э.н.п. для $x$ в конусе $H$ тогда и только тогда, когда $h_{0}-$ э.н.п. для $x$ в расширенном конусе $H_{J\left(h_{0}\right)}$.

Лемма 1.2. Пусть $x \in L[a ; b]$ и $h_{0} \in H$. Следующие условия эквивалентны:

1. $\int_{a}^{b}\left(h(t)-h_{0}(t)\right) \operatorname{sign}\left(x(t)-h_{0}(t)\right) d t \leq 0$ для любого $h \in H$;

2. $\int_{a}^{b} \varphi(t) \operatorname{sign}\left(x(t)-h_{0}(t)\right) d t \leq 0$ для любого $\varphi \in H_{J\left(h_{0}\right)}$;

3. $\int_{a}^{b} h_{0}(t) \operatorname{sign}\left(x(t)-h_{0}(t)\right) d t=\sup _{h \in H} \int_{a}^{b} h(t) \operatorname{sign}\left(x(t)-h_{0}(t)\right) d t$;

4. $\int_{a}^{b} h_{0}(t) \operatorname{sign}\left(x(t)-h_{0}(t)\right) d t=0$;

$\int_{a}^{b} h(t) \operatorname{sign}\left(x(t)-h_{0}(t)\right) d t \leq 0$ для любого $h \in H$.

Доказательство. Импликации $(1) \Rightarrow(2) \Rightarrow(3) \Rightarrow(1)$ очевидны. Покажем, что $(2) \Leftrightarrow(4)$. Предположим в начале, что

$$
\int_{a}^{b} \varphi(t) \operatorname{sign}\left(x(t)-h_{0}(t)\right) d t \leq 0 \text { для любого } \varphi \in H_{J\left(h_{0}\right)} .
$$

Тогда из условия $h_{0},-h_{0} \in H_{J\left(h_{0}\right)}$ следует

$$
\int_{a}^{b} h_{0}(t) \operatorname{sign}\left(x(t)-h_{0}(t)\right) d t=0 .
$$

Далее, для каждого $h \in H$ элемент $h-h_{0} \in H_{J\left(h_{0}\right)}$ и поэтому

$$
\int_{a}^{b} h(t) \operatorname{sign}\left(x(t)-h_{0}(t)\right) d t=\int_{a}^{b}\left(h(t)-h_{0}(t)\right) \operatorname{sign}\left(x(t)-h_{0}(t)\right) d t \leq 0 .
$$

Таким образом, импликация $(2) \Rightarrow(4)$ верна.

Покажем теперь справедливость импликации $(4) \Rightarrow(2)$. Пусть $\varphi \in H_{J\left(h_{0}\right)}$. Тогда по лемме 1.1 найдутся $\alpha \geq 0$ и $h \in H$ такие, что

$$
\varphi=\alpha\left(h-h_{0}\right) \text {. }
$$

Следовательно

$$
\begin{gathered}
\int_{a}^{b} \varphi \operatorname{sign}\left(x(t)-h_{0}(t)\right) d t= \\
=\int_{a}^{b} \alpha\left(h(t)-h_{0}(t)\right) \operatorname{sign}\left(x(t)-h_{0}(t)\right) d t= \\
=\alpha \int_{a}^{b} h(t) \operatorname{sign}\left(x(t)-h_{0}(t)\right) d t \leq 0 .
\end{gathered}
$$

Итак, лемма доказана полностью. 
В силу следствия 1.1 каждое из условий (1)-(4) леммы 1.2 является достаточным, а в случае, когда множество

$$
\left\{t \in[a ; b]: x(t)=h_{0}(t)\right\}
$$

имеет меру нуль и необходимо, для того чтобы элемент $h_{0}$ был э.н.п. для функции $x \in L[a ; b] \backslash H$ в конусе $H$, при этом

$$
E(x, H)=\left\|x-h_{0}\right\|=\int_{a}^{b} x(t) \operatorname{sign}\left(x(t)-h_{0}(t)\right) d t .
$$

Подводя итог вышесказанному, сформулируем следующую теорему.

Теорема 1.3. Пусть конус $H$ имеет вид

$$
H=\left\{\sum_{k=1}^{n} \alpha_{k} \varphi_{k}: \alpha_{k} \in R, \alpha_{j} \geq 0, k>m\right\},
$$

$h_{0} \in H, H_{1}$ - подпространство, содержащееся в конусе $H$, базисом которого являются функции множества $\left\{\varphi_{k}: k \in J\left(h_{0}\right)\right\}$, т.е.

$$
H_{1}=\left\{\sum_{k \in J\left(h_{0}\right)} \alpha_{k} \varphi_{k}: \alpha_{k} \in R\right\} .
$$

Для того чтобы $h_{0}$ являлся э.н.п. для $x \in L[a ; b] \backslash H$ при аппроксимации конусом H, достаточно, а в случае, когда множество

$$
\left\{t \in[a ; b]: x(t)=h_{0}(t)\right\}
$$

имеет нулевую меру и необходимо, выполнение следующих условий:

1) $\int_{a}^{b} \varphi(t) \operatorname{sign}\left(x(t)-h_{0}(t)\right) d t=0$ для любого $\varphi \in H_{1}$;

2) $\int_{a}^{b} h(t) \operatorname{sign}\left(x(t)-h_{0}(t)\right) d t \leq 0$ для любого $h \in H$,

при этом

$$
\left\|x-h_{0}\right\|=E(x, H)=E\left(x, H_{1}\right)=E\left(x, H_{J\left(h_{0}\right)}\right) .
$$

Доказательство. Доказательство. Достаточность очевидна, т.к. $h_{0} \in H_{1}$. Необходимость вытекает из следствия 1.1 и леммы 1.2.

Так как

$$
h_{0} \in H_{1}, \quad H_{1} \subset H_{J\left(h_{0}\right)},
$$

то в случае, когда $h_{0}$ является э.н.п. для $x$ в конусе $H$, получим

$$
\left\|x-h_{0}\right\|=E(x, H) \geq E\left(x, H_{1}\right) \geq E\left(x, H_{J\left(h_{0}\right)}\right)=\left\|x-h_{0}\right\|,
$$

при этом равенство $E\left(x, H_{J\left(h_{0}\right)}\right)=\left\|x-h_{0}\right\|$ следует из теоремы 1.2 . Итак, теорема верна. 


\section{2. Аналог теоремы Маркова}

Пусть $t_{1}, t_{2}, \ldots, t_{r}$ есть различные точки отрезка $[a ; b]$, функция

$$
\omega(t)=\left(t-t_{1}\right)\left(t-t_{2}\right) \ldots\left(t-t_{r}\right), t \in[a ; b] .
$$

Будем говорить, что точки $t_{1}, t_{2}, \ldots, t_{r}$ - все точки перемены знака функции $\varphi \in L[a ; b]$ на отрезке $[a ; b]$, если, либо почти всюду на $[a ; b]$ выполняется равенство

$$
\operatorname{sign} \varphi(t)=\operatorname{sign} \omega(t)
$$

либо почти всюду на $[a ; b]$ имеет место

$$
\operatorname{sign} \varphi(t)=-\operatorname{sign} \omega(t)
$$

Теорема 2.1. Пусть $\left\{\varphi_{1}, \varphi_{2}, \ldots, \varphi_{n}\right\}$ - линейно независимая система в $L[a ; b]$, $m$ - целое неотрицательное число. Конус $H$ имеет вид

$$
H=\left\{\sum_{k=1}^{n} \alpha_{k} \varphi_{k}: \alpha_{k} \in R, \alpha_{j} \geq 0, k>m\right\}
$$

функция $g \in L[a ; b]$ такова, что:

a) $t_{1}, t_{2}, \ldots, t_{r}$ - все точки перемены знака функции $g$;

b) $\int_{a}^{b} h(t) \operatorname{sign} g(t) d t \leq 0$ для любого $h \in H$.

Если $x \in L[a ; b] \backslash H$ и $h_{0} \in H$ обладают свойствами:

1) $t_{1}, t_{2}, \ldots, t_{r}-$ все точки перемены знака для $x-h_{0}$;

2) $\int_{a}^{b} h_{0}(t)$ sign $g(t) d t=0$,

то $h_{0}$ - э.н.п. для функции $x$ в конусе $H$ и

$$
E(x, H)=\left|\int_{a}^{b} x(t) \operatorname{sign} g(t) d t\right|
$$

Доказательство. Доказательство. В силу следствия 1.1 и леммы 1.2 получаем, что $h_{0}$ есть э.н.п. для $x$ в конусе $H$. Тогда

$$
E(x, H)=\int_{a}^{b} x(t) \operatorname{sign}\left(x(t)-h_{0}(t)\right) d t=\left|\int_{a}^{b} x(t) \operatorname{sign} g(t) d t\right|
$$

и теорема верна. 
Если конус $H$ является подпространством конечной размерности, то получим известный результат А.А. Маркова [2].

Теорема 2.2 (Марков А.А.) Пусть

$$
H=\left\{\sum_{k=1}^{n} \alpha_{k} \varphi_{k}: \alpha_{k} \in R\right\}
$$

функция $g \in L[a ; b]$ такова, что
a) $t_{1}, t_{2}, \ldots, t_{r}-$ все точки перемены знака функции $g$;
b) $\int_{a}^{b} h(t) \operatorname{signg}(t) d t=0$ для любого $h \in H$.

Если функция $x \in L[a ; b] \backslash H$ и элемент $h_{0} \in H$ таковы, что $t_{1}, t_{2}, \ldots, t_{r}-$ все точки перемены знака $x-h_{0}$, то $h_{0}$ есть э.н.п. функции $x$ в конечномерном подпространстве $H$ и

$$
E(x, H)=\left|\int_{a}^{b} x(t) \operatorname{sign} g(t) d t\right|
$$

\section{3. Способы построения наилучших приближений при аппроксимации тригонометрическими и алгебраическими полиномами}

Особенностью теоремы Маркова А.А. и ее аналога является то, что функция $g$, удовлетворяющая соотношениям (a), (b), строится независимо от приближаемой функции $x$. Она зависит лишь от выбранного подпространства $H$.

В [2] указаны соответствующие функции $g$ для случаев приближения подпространствами тригонометрических и алгебраических полиномов и указаны способы построения элементов наилучшего приближения.

Рассмотрим отмеченные способы построения элементов наилучшего приближения при аппроксимации тригонометрическими и алгебраическими полиномами.

Лемма 3.1. При любых $\alpha$ и $a \in R$ для тригонометрического полинома $T_{n}$ порядка не выше $n$ выполняется равенство

$$
\int_{a}^{a+\pi} T_{n}(t) \operatorname{sign} \sin [(n+1)(t-\alpha)] d t=0 .
$$

Обозначим через $L_{2 \pi}$ пространство $2 \pi-$ периодических функций, суммируемых на каждом конечном промежутке $[a ; a+2 \pi]$, с нормой

$$
\|f\|=\int_{a}^{a+2 \pi}|f(t)| d t=\int_{0}^{2 \pi}|f(t)| d t .
$$


Если рассмотреть функции из $L_{2 \pi}$ лишь на промежутке $[a ; a+2 \pi]$, то сразу получим, что это пространство линейно изометрично $L[a ; a+2 \pi]$. Через $L_{2 n+1}$ обозначим подпространство тригонометрических полиномов порядка не выше $n$. Пусть

$$
\begin{gathered}
h_{1}(t)=1, h_{2}(t)=\sin t, h_{3}(t)=\cos t, \ldots, h_{2 n}(t)=\sin (n t), h_{2 n+1}(t)=\cos (n t), \\
H=\left\{\sum_{k=1}^{2 n+1} \alpha_{k} h_{k}: \alpha_{k} \in R, \alpha_{j} \geq 0, k>m\right\},
\end{gathered}
$$

где $m$ - целое неотрицательное число. Из приведенной леммы 3.1, теоремы Маркова А.А. и теоремы 2.1 немедленно вытекает следующее утверждение.

Теорема 3.1. Пусть $f \in L_{2 \pi}, T_{n}$ - тригонометрический полином порядка не выше $n$ принадлежит конусу $H \subset L_{2 n+1}$. Если $t_{k}=\alpha+\frac{k \pi}{(n+1)}, k=0,1, \ldots, 2 n+1$, суть все точки перемены знака разности $f-T_{n}$ на некотором промежутке $(a ; a+2 \pi)$, то $T_{n}$ - э.н.п. функции $f$ в подпространстве $L_{2 n+1}$ и в конусе $H$, при этом

$$
E\left(f ; L_{2 n+1}\right)=E(f ; H)=\left|\int_{-\pi}^{\pi} f(t) \operatorname{sign} \sin [(n+1)(t-\alpha)] d t\right| .
$$

Доказательство. Используя линейную изометрию указанных пространств и полагая $g(t)=\sin [(n+1)(t-\alpha)]$, получим, что точки

$$
t_{0}, t_{1}, t_{2}, \ldots, t_{2 n+1}
$$

- все точки перемены знака функций $g$ и $f-T_{n}$ на любом открытом промежутке длиной $2 \pi$, который их содержит. Из теоремы Маркова А.А. и ее аналога получим требуемое.

Лемма 3.2. Для любого алгебраического полинома $P_{n}$ степени не выше $n$ выполняется равенство

$$
J=\int_{-1}^{1} P_{n}(t) \operatorname{sign} \sin [(n+2) \arccos t] d t=0 .
$$

Если рассмотреть пространство $L[-1 ; 1]$, его подпространство $L_{n}$, состоящее из всех полиномов степени не выше $n$, и конус

$$
H=\left\{\sum_{k=0}^{n} \alpha_{k} t^{k}: t \in[-1 ; 1], \alpha_{k} \in R, \alpha_{j} \geq 0, k>m\right\},
$$

где $m$ - целое неотрицательное число, то условие (b) теоремы Маркова А.А., а также условия (b) и (2) теоремы 2.1, будут выполнены для функции

$$
g(t)=\sin [(n+2) \arccos t] .
$$


Так как все точки перемены знака этой функции на $(-1 ; 1)$ исчерпываются точками

$$
t_{k}=\cos \frac{k \pi}{n+2}, k=1,2, \ldots, n+1,
$$

то справедлива следующая

Теорема 3.2. Пусть $f \in L[-1 ; 1], P_{n}$ - полином степени не выше $n$ принадлежит конусу $H \subset L_{n}$. Если точки

$$
t_{k}=\cos \frac{k \pi}{n+2}, k=1,2, \ldots, n+1,
$$

- это все точки перемены знака разности $f-P_{n}$ на $(-1 ; 1)$, то $P_{n}$ есть э.н.п. функции $f$ в подпространстве $L_{n}$ и в конусе $H$, причем

$$
E\left(f ; L_{n}\right)=E(f ; H)=\left|\int_{-\pi}^{\pi} f(t) \operatorname{sign} \sin [(n+2) \arccos t] d t\right| .
$$

В случае аппроксимации подпространством $L_{n}$ теорема 3.2 допускает следующее применение. Пусть $f \in C[-1 ; 1]$ и требуется построить ее полином наилучшего приближения ( в метрике $L[-1 ; 1]$ ) степени не выше $n$. По узлам $t_{k}$, указанным в теореме 3.2 , построим интерполяционный полином $P_{n}$ для функции $f$. Если окажется, что они являются точками перемены знака $f-P_{n}$ и других точек перемены знака нет, то $P_{n}-$ э.н.п. функции $f$ в $L_{n}$.

В периодическом случае при аппроксимации подпространством тригонометрических полиномов порядка не выше $n$ подобное применение теоремы 3.1 несколько сложнее, поскольку число точек $t_{k}$ равно $(2 n+2)$ и на единицу больше, чем требуется для разрешимости интерполяционной задачи. Однако, здесь в нашем распоряжении имеется еще один дополнительный параметр - число $\alpha$.

\section{Заключение}

Полученные результаты носят законченный характер и могут быть использованы в различных задачах прикладных направлений.

\section{Список литературы}

[1] Дрожжин И.А. Наилучшие приближения суммируемых функций элементами замкнутого выпуклого множества с ограничениями // Применение функционального анализа в теории приближений. 2014. № 35. С. 55-62.

[2] Даугавет И.К. Введение в теорию приближения функций. Л.: ЛГУ, 1977.

[3] Корнейчук Н.П. Экстремальные задачи теории приближений. М.: Наука, 1976.

[4] Peisker P. An alternant criterion for Haar cones // Journal of Approximation Theory. 1983. Vol. 37, № 3. Pp. 262-268. https://doi.org/10.1016/0021-9045(83) 90052-7 


\section{Образец цитирования}

Дрожжин И.А. Аналог теоремы Маркова // Вестник ТвГУ. Серия: Прикладная математика. 2017. № 4. C. 73-83. https://doi.org/10.26456/vtpmk190

\section{Сведения об авторах}

\section{1. Дрожжин Игорь Александрович}

доцент кафедры математического анализа Тверского государственного университета.

Россия, 170100, г. Тверь, ул. Желябова, д. З3, ТвГУ. 


\title{
AN ANALOG OF MARKOV'S THEOREM
}

\author{
Drozhzhin Igor Alexandrovich \\ Associate professor at Mathematical Analysis department, \\ Tver State University \\ Russia, 170100, Tver, 33 Zhelyabova str., TverSU.
}

\begin{abstract}
Received 16.06.2017, revised 17.07.2017.
In the space of functions summable on a segment, the sign of the best Approximation for approximation by elements of a finite-dimensional cone, which is Analogue of the well-known theorem of Markov A.A.

Keywords: banach space, closed convex set, summable function, best approximation, cone of finite dimension, extended cone, interpolation polynomial.
\end{abstract}

\section{Citation}

Drozhzhin I.A. An analog of Markov's theorem. Vestnik TvGU. Seriya: Prikladnaya Matematika [Herald of Tver State University. Series: Applied Mathematics], 2017, no. 4, pp. 73-83. (in Russian). https://doi.org/10.26456/vtpmk190

\section{References}

[1] Drozhzhin I.A. Best approximations of summable functions by elements of a closed convex set with constraints. Primenenie Funktsional'nogo Analiza v Teorii Priblizhenii [Application of Functional Analysis in Approximation Theory], 2014, no. 35, pp. 55-62. (in Russian)

[2] Daugavet I.K. Vvedenie v Teoriju Priblizhenija Funkcij [Introduction to the Theory of Approximation of Functions]. LGU Publ., Leningrad, 1977. (in Russian)

[3] Kornejchuk N.P. Ekstremalnye Zadachi Teorii Priblizhenij [Extremal Problems in Approximation Theory]. Nauka Publ., Moscow, 1976. (in Russian)

[4] Peisker P. An alternant criterion for Haar cones // Journal of Approximation Theory. 1983. Vol. 37, № 3. Pp. 262-268. https://doi.org/10.1016/0021-9045(83) 90052-7 